\title{
Acoustical Society of America
}

\section{The Journal of the Acoustical Society of America}

The communicative influence of gesture and action during speech comprehension: gestures have the upper hand

Spencer Kelly ${ }^{1}$, Meghan Healey ${ }^{2}$, Asli Ozyurek ${ }^{3}$ and Judith Holler ${ }^{4}$

J. Acoust. Soc. Am. 131, 3311 (2012); http://dx.doi.org/10.1121/1.4708385

\section{Abstract}

Hand gestures combine with speech to form a single integrated system of meaning during language comprehension (Kelly et al., 2010). However, it is unknown whether gesture is uniquely integrated with speech or is processed like any other manual action. Thirty-one participants watched videos presenting speech with gestures or manual actions on objects. The relationship between the speech and gesture/action was either complementary (e.g., "He found the answer," while producing a calculating gesture vs. actually using a calculator) or incongruent (e.g., the same sentence paired witl the incongruent gesture/action of stirring with a spoon). Participants watched the video (prime) and then responded to a written word (target) that was or was not spoken in the video prime (e.g., "found" or "cut"). ERPs were taken to the primes (time-locked to the spoken verb, e.g., "found") and the written targets. For primes, there was a larger frontal N400 (semantic processing) to incongruent vs. congruent items for the gesture, but not action, condition. For targets, the P2 (phonemic processing) was smaller for target words following congruent vs. incongruent gesture, but not action, primes. These findings suggest that hand gestures are integrated with speech in a privileged fashion compared to manual actions on objects.

(C) 2012 Acoustical Society of America

DOII: http://dx.doi.org/10.1121/1.4708385

\section{Key Topics}

Speech

Speech processing

systems

\section{Most read this month}

Evaluation of smartphone sound measurement applications ${ }^{a)}$

Chucri A. Kardous and Peter B. Shaw

Coffee roasting acoustics 


\section{Preston S. Wilson}

Exploiting the leaky-wave properties of transmission-line metamaterials for single-microphone direction finding

Hussein Esfahlani, Sami Karkar, Hervé Lissek and Juan R. Mosig

\section{Most cited this month}

Theory of Propagation of Elastic Waves in a Fluid-Saturated Porous Solid. I. Low-Frequency Range

M. A. Biot

Transformed Up-Down Methods in Psychoacoustics

H. Levitt

Theory of Propagation of Elastic Waves in a Fluid-Saturated Porous Solid. II. Higher Frequency Range

M. A. Biot

\section{Access Key}

\section{FFree Content}

OAOpen Access Content

SSubscribed Content

TFree Trial Content

/content/realmedia?fmt=ahah\&adPositionList=

\&advertTargetUrl=//oascentral.aip.org/RealMedia/ads/\&sitePageValue=asadl.org/jasa/131/4/10.1121 /1.4708385\&pageURL=http://scitation.aip.org/content/asa/journal/jasa/131/4/10.1121/1.4708385'

Right1,Right2,Right3, 